\title{
A Comparison of Acyltransferase Activities in vitro with the Distri- bution of Fatty Acids in Lecithins and Triglycerides in Vivo
}

\author{
W. E. M. Lands, University of Michigan, Ann Arbor, Michigan; M. L. Blank, L. J. Nutter and O. S.
}

Privett, The University of Minnesota, Austin, Minnesota

\begin{abstract}
The location and configuration of a double bond in a fatty acid influences the rate of its acyltransferase-catalyzed esterification to form lecithin and its distribution in vivo between the primary and secondary positions of triglycerides and lecithins.

Saturated acids of shorter chain length are transferred at rates similar to the long chain unsaturated acids.

The positional distributions of acids in the diglyceride units of liver triglycerides appear to be similar to that found in the lecithins.

Acyltransferase activities measured in vitro have a considerable predictive value in terms of the ultimate distribution of fatty acids in glycerolipids in vivo.
\end{abstract}

\section{INTRODUCTION}

$\mathrm{M}$ UCH OF THE RECENT WORK with acyltransferases supports the general concept that their activity may be important in controlling the fatty acid composition of tissue phosphoglycerides. The extent to which other reactions may control this composition is not clear, and it is evident that a series of enzymatic factors exist, all of which may exert some effect. Attempts to show selective esterifications in forming phosphatidic acid (1) were not as successful as those involving lecithin formation (2). Thus, the current evidence suggests that the characteristic distribution of fatty acids between the 1- and 2-positions may arise in part from a redistribution of acids after the nitrogenous base has been attached to the molecule. Further work has emphasized that the classification of fatty acids as either saturated or unsaturated is an unsatisfactory oversimplification (3) when describing their metabolic properties. Rather, each fatty acid should be regarded as having a characteristic metabolic fate.

This concept led us to consider the fate of unnatural fatty acids as well as a series of natural acids while testing the selectivity of the acyltransferases. A series of $c$ is and trans isomers of octadecadienoate was used since three of the four acids are not encountered in normal metabolic processes. A comparison of the distribution of these acids in vivo with the relative esterification rates in vitro indicates the degree to which acyltransferase specificities could control the distribution of a fatty acid. Our results show that the enzymatic activities measured in vitro have a considerable value in predicting the ultimate distribution of fatty acids in glycerolipids in vivo.

\section{EXPERIMENTAL}

\section{Synthesis of Acyl-CoA Derivatives}

The procedure described by Seubert (4) was used to synthesize several saturated long-chain acyl-CoA esters. The derivatives of the polyunsaturated acids, however, are unstable in dilute aqueous solutions and the modifications described below provided high yields of thiol esters that were $100 \%$ active in the acyltransferase systems.

All solvents used in the following procedure contained $0.1-0.5 \%$ 1,2-dihydro-6-ethoxy-2,2,4trimethylquinoline (Santoquin, ethoxyquin) as an antioxidant. ${ }^{1}$ Tetrahydrofuran was distilled from $\mathrm{LiAlH}_{4}$ immediately before use and all aqueous solutions were freshly prepared using water saturated with Santoquin. The reaction was carried out in a $50 \mathrm{ml}$ Erlenmeyer flask and was stirred continously with a magnetic stirrer.

Coenzyme A $(100 \mathrm{mg})$ was dissolved in 30 $\mathrm{ml}$ of aqueous tetrahydrofuran $(50 \%)$, and the $\mathrm{pH}$ was adjusted to 7.5-8.0 with $1 N$ $\mathrm{NaOH}$. The mercaptan content was estimated by removing $0.1 \mathrm{ml}$ of the reaction mixture and adding it to a solution of 5,5'-dithio-bis-(2nitrobenzoic acid) (DTN) in phosphate buffer $(\mathrm{pH} 7.4)$ (5).

After the $\mathrm{pH}$ and mercaptan content of the solution were determined, $100 \mathrm{mg}$ of the acylchloride was added and the $\mathrm{pH}$ readjusted to 7.5-8.0 with dropwise addition of $\mathrm{NaOH}$. The mercaptan content was checked, and the addition of acylchloride and $\mathrm{NaOH}$ was continued until no mercaptan could be detected. At this point the reaction mixture was allowed to stand for $5 \mathrm{~min}$ and then $1 \mathrm{~g}$ of stearic acid was added to facilitate isolation of the less crystalline, unsaturated acyl-CoA esters in the subsequent steps.

1 Antioxidant recommended by R. T. Holman and supplied by the Monsanto Co. 
The reaction mixture was placed in a $100 \mathrm{mg}$ round flask, chilled on ice, and $10 \mathrm{ml}$ of $10 \%$ $\mathrm{HClO}_{1}$ was added. The tetrahydrofuran was removed using a rotary evaporator, and the white precipitate was collected on a Buchner funnel while avoiding excessive exposure to $0_{2}$. The solid was transferred to centrifuge tubes and washed thoroughly with ether to remove fatty acids. The residue of thiol ester was dissolved in $8 \mathrm{ml}$ of cold water, the $\mathrm{pH}$ adjusted to 5.5 with a few drops of $\mathrm{NaHCO}_{3}$, and the insoluble material (cellulose fibers, etc.) removed by centrifugation. The $\mathrm{CoA}$ thiol ester was reprecipitated by adding $2 \mathrm{ml}$ of $10 \% \mathrm{HClO}_{4}$, collected by centrifugation, and washed with ether as before.

The yield was generally 60 to $70 \mu$ moles of acyl-CoA per $100 \mu$ moles of coenzyme $A$ used as starting material. Santoquin interferes with the usual $232 / 260$ assay for acyl-CoA (4), but the phosphorus content was measured (6) and compared to the extent of coenzyme A released (5) with acyltransferase systems to indicate that the material was $100 \%$ reactive (2).

\section{Enzymatic Studies}

The acyltransferase rates were determined spectrophotometrically under conditions of maximal velocity using DTN to measure CoA release as described earlier (2). The isomeric acylglycerol phosphorylcholine substrates were prepared by selective hydrolyses of lecithin and plasmalogen (3). Preparations of the 2-acylisomer generally required higher levels to saturate the enzyme than did the 1-acyl-isomer. The specific activities reported in this paper are the averages of three separate experiments with rat liver microsomes. The rates have been corrected for the small amount of hydrolase activity $(+1.8 \mathrm{~m} \mu$ moles $/ \mathrm{min} / \mathrm{mg}$ protein $)$ noted in the controls. In addition, all reaction mixtures containing the 2-acyl-GPC ${ }^{2}$ exhibited a very slow and consistent decrease in absorbance regardless of initial enzyme or acyl-CoA levels. The rate of decrease was proportional to the amount of the 2-acyl-GPC added and appeared to be due to traces of acetone-soluble compounds that reacted slowly with the yellowcolored arylmercaptan. Under the conditions used, the two corrections required for these reaction mixtures cancelled each other.

\section{Feeding Experiments}

In these studies, male rats of the SpragueDawley strain were made deficient in essential fatty aeids by maintaining them from weaning to 4 months of age on a fat-free diet as described in previous work from this laboratory (8). Groups of a minimum of 5 animals each were then fed (as a supplement to the basic fat-free diet) $5 \%$ by weight of either cis-9, cis-12, cis-9,trans-12- or trans-9,trans-12isomers of methyl linoleate for 18 to 20 days. Th? animals were killed, the livers excised, quisk frozen on dry ice and stored at $-20 \mathrm{C}$. The lipid fraction was recovered by extraction with chloroform-methanol as previously deseribed (8). The triglycerides were isolated by a combination of DEAE-cellulose and thin-layer chromatography (TLC) and the lecithins were isolated by a combination of DEAE-cellulose and ammonium silicate chromatography (9). The purity of the lecithins was confirmed by thin-layer chromatography and infrared analysis.

\section{Positional Analyses of the Lecithins and Triglycerides}

The lecithins were hydrolyzed with Ophiogaphus hannah (Ross Allen Reptile Institute, Silver Springs, Florida) phospholipase A, using conditions deseribed by Robertson and Lands (10). After the hydrolysis was complete, as determined by periodic analysis with TLC, the products were isolated by preparative TLC. The fatty acid and 1-acyl-GPC fractions were separated by TLC on plates coated with chloroform-extracted Silica Gel G (A. G. Merek, Darmstad, Germany) using chloroform/ methanol/water $(70 / 30 / 3)$. The spots were detected by spraying the plate with $2^{\prime}, 7^{\prime}$ dichlorofluorescein and viewing it under ultraviolet light. The spots were seraped into test tubes with constricted necks for sealing. About $3 \mathrm{ml}$ of methanol containing $6 \%(\mathrm{~W} / \mathrm{W})$ of dry $\mathrm{HCl}$ was added to each tube. The tubes were then flushed with nitrogen, sealed with a torch and heated in a boiling water bath to convert the sample to methyl esters.

The methyl esters were recovered and analyzed by gas-liquid chromatography using a F \& M Model 609 hydrogen flame ionization gas chromatograph equipped with a $7 \mathrm{ft} \times$ $1 / 4$ in. column packed with ethylene glycol succinate polyester phase (EGSS-X, Applied Science Laboratories) on Chromosorb W, 100200 mesh at $185 \mathrm{C}$. The fatty acid composition was calculated directly from the proportionalities of the peak areas and expressed as weight percent. The conditions for linearity of detector response were determined with stan-

\footnotetext{
2 The stereospecific assignment described by Hirschmann (7) is used to indicate the stereochemistry of the glycerides described in this paper. Glycerol-3-phosphorylcholine will be abbreviated as GPO.
} 
dard mixtures of methyl esters obtained frou The Hormel Institute.

Under the conditions employed, the geometric isomers of linoleate could not be separated from each other. However, since the animals were starved of lipid for four months the triglycerides and lecithins were virtually devoid of the naturally-occurring isomer of linoleic acid. Thus, 18:2 analyzed in each experiment may be considered to consist of the isomer of linoleic acid that was fed. That dietary trans, trans linoleic acid (linoelaidic acid) was deposited in the lipids of rats was demonstrated by a direct determination in a previous study (8).

The acids liberated by phospholipase $A$ represent those originally esterified at the 2position and the acyl-GPC content indicates the composition at the 1-position. The ratio of the percentage content of an acid at the 2- and 1-positions was then calculated. This ratio and the total percentage content of the acid in the total lecithins provide a fairly detailed description of the distribution of fatty acids in lecithins using only two values for each acid.

The distribution of the fatty acids in the 2-position of triglycerides was determined with pancreatic lipase under the conditions described by Mattson and Volpenhein (11) scaled down for use on a sample of about one-tenth the size employed by these workers. In this method the hydrolysis was stopped at about $50 \%$ completion. The monoglycerides obtained as products of the reaction were isolated from the free acids, di- and triglycerides by TLC. The fatty acid composition of the 2-monoglycerides was determined by forming methyl esters as described above. Since this lipase method does not allow distinction between the composition of the 1 - and 3-positions, a ratio representing the distribution between the 2 and the 1-position was calculated using the average value for the primary positions. Thus the estimated $2 / 1$ ratio (secondary/primary positions) for a given acid in the triglycerides is as follows.

$2 \times(\%$ content in monoglycerides)

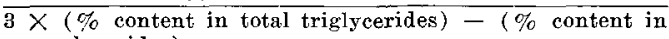
monoglycerides)

In this way the distribution and composition of fatty acids in the triglycerides can be described using: only two values similar to those used for the lecithins described above.

\section{RESULTS}

The results in Table I show that the acyltransferases can discriminate between the
TABLE I

Acyltransferase Specificities of Rat Liver Microsomes

The reaction mixtures contained 25 mumoles of acyl-

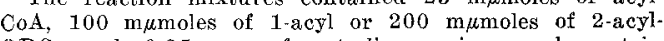
$G P C$, and $0.25 \mathrm{mg}$ of rat liver microsomal protein (treated with DFP (2)) in a final rolume of $1.0 \mathrm{ml}$ (see Experimental section).

\begin{tabular}{|c|c|c|c|}
\hline \multirow[b]{2}{*}{ Acyl-CoA ${ }^{a}$} & \multicolumn{2}{|c|}{ Position acylated } & \multirow[b]{2}{*}{ 2/1 Ratio } \\
\hline & No. 2 & No. 1 & \\
\hline \multicolumn{4}{|c|}{$\mathrm{m} \mu \mathrm{moles} / \mathrm{min} / \mathrm{mg}$ protein } \\
\hline $18: 0$ & 1.7 & 19.6 & 0.087 \\
\hline $16: 0$ & 3.5 & 20.3 & 0.17 \\
\hline $14: 0$ & 4.3 & 7.6 & 0.56 \\
\hline $12: 0$ & 6.5 & 7.0 & 0.93 \\
\hline $16: 1-\mathrm{e} 9$ & 6,0 & 0.4 & 16.2 \\
\hline $18: 1-c 9$ & 12.4 & 3.9 & 3.2 \\
\hline $18: 1-\mathrm{t} 9$ & 10.3 & 23.3 & 0.44 \\
\hline $18: 2-\mathrm{c} 9, \mathrm{c} 12$ & 23.1 & 1,8 & 12.9 \\
\hline $18: 2-c 9, \mathrm{t}, 12$ & 9.1 & 2.0 & 4.6 \\
\hline $18: 2-\mathrm{t} 9, \mathrm{c} 12$ & 13.7 & 13.8 & 1.0 \\
\hline $18: 2-\mathrm{t} 9, \mathrm{t} 12$ & 6.1 & 28.4 & 0.21 \\
\hline $18: 3$-all $\mathrm{e}$ & 14.6 & 1.8 & 8.0 \\
\hline $20: 4-$ all c & 19.6 & 2.3 & 8.5 \\
\hline
\end{tabular}

a The number notations indicate the chain length and number of double bonds, with the configuration and position indicated after the hyphen (e.g., elaidate is 18:1-t9).

normal eis isomers and the unnatural trans forms. The trans-9,trans-12-isomer was esterified rapidly at the 1-position. When a cis configuration was present (in the trans-9,cis-12isomer) the rate of esterification at the 1position of 2-acyl-GPC was considerably diminished. When the cis configuration was in the 9-position, it interfered even more seriously with that acylation. The effect of configuration was not as marked on the rates of reaction at the 2-position although the trans dienoates reacted significantly less rapidly than the cis isomers. For each fatty acid considered in Table $I$, the enzymatic specific activities determined give different ratios for the rates of esterification at the 2- and 1positions. In the case of the dienoates, the ratios extend over a 60 -fold range from the all-cis to the all-trans isomers.

The effect of decreasing chain length in the naturally-occurring saturated acids was a decrease in the rate of esterification at the 1 position with an increased rate of reaction at the 2-position. This is in agreement with earlier results (3) which indicated that some saturated acids of shorter chain length are handled like the unsaturated long chain acids. The reactivity found for 16:1 at the 1-position was particularly low, making the $2 / 1$ ratio markedly higher than, for example, that found for 18:1. The low activity was ehecked many times but it consistently remained lower than that for any other acid tested. If the activity for reaction at the 1 -position were approxi- 
wately $2 \mathrm{~m} \mu \mathrm{moles} / \mathrm{min} / \mathrm{mg}$ protein, the $2 / 1$ ratio would be 3 , and thus closely resemble the results with 18:1.

The relative pattern of activities for the various acids in Table $I$ has also been noted in this laboratory with the microsomal fraetion of livers from rats that had been subjected to a variety of diets deficient in fatty acids such as starvation, fat-free and long-term essential fatty acid deficiency. The only noticeable difference from the values reported here was an elevated specific activity (per mg protein) for all acids tested with the enzymes prepared from rats with a severe essential fatty acid deficiency. However, in this ease, the relative pattern of activities still remained the same. The observed distribution of octadecadienoate between the secondary and primary positions differed according to the isomer which was fed (Table II). The cis,cis-isomer was found predominantly at the 2-position, the trans,trans at the 1-position, and the cis,transisomer gave values intermediate between those two. Although the gas ehromatographie method used to measure the acids in this work did not distinguish between the different geometrical isomers, the earlier results of Privett and Blank (8) indicate that isomers other than that added to the diet were not present to any appreciable extent. The calculated $2 / 1$ ratios for the in vivo distribution of acids in the lecithins cover a wide range of values which is in interesting agreement with that found for the in vitro acyltransferase rates.

The positional distribution ratio for the trans,trans-isomer was very similar for both the lecithins and triglycerides. The distribution ratios for palmitate in vivo (given in Table II) were very similar to those found for the in vitro acylation rates, and were similar in both the lecithins and triglycerides. This result was not generally noted for those acids which are predominantly found at the 2-position (ratios greater than 1). Octadecenoate, for instance, gave values from 1.2 to 2.1 for the triglycerides and 0.8 to 2.5 for the lecithins. When the diet contained linoleate as a supplement, the resultant 18:1 content of the liver lecithin (see Table III) was only 9\%, and a low value (0.8) (see Table II) was obtained for the $2 / 1$ distribution of $18: 1$ in the lecithins. In these animals, arachidonate accounted for $33 \%$ of the total acids and was located almost exclusively at the 2-position. When the diet contained low levels of cis double bonds (fat free, cis,trans, or trans,trans) the content of monoenoie aeids in the tissue lipids rose, particularly that of $16: 1$. In these cases the rise in monoenoate content oceurred primarily at the 2-position, whereas the arachidonate content fell, and the $2 / 1$ distribution ratio for the monoenoates in vivo approached values closer to the in vitro results.

\section{DISCUSSION}

In enzymatic studies with rat liver microsomes, the trans double bond seems similar to a saturated carbon chain in that the cis-9,trans-12dienoate most closely resembles the cis-9monoeneoate in acylation rates, whereas the trans -9 , trans-12-dienoate most closely resembles the trans-9-monoeneoate. Also, esterification at the 2-position does not seem to be as sensitive to configurational differences as that at the 1position which is in agreement with earlier results (3). The latter position is only slowly esterified by acids containing a cis-9 configuration. Additional comparisons of these acids suggest that at the 1-position the most linear trans configuration is slightly preferred even to the saturated chain. The ability of the cis-9 configuration to diminish this reaction rate more effectively than the cis-12 raises an interesting question of the effect of other positional isomers. The $\Delta 9$-desaturase system in rat

TABLE II

Positional Distribution of Fatty Acids in Rat Liver Lipidsa

\begin{tabular}{|c|c|c|c|c|c|c|}
\hline \multirow{2}{*}{$\begin{array}{c}\text { Dietary } \\
\text { supplement }\end{array}$} & \multirow{2}{*}{$\begin{array}{l}\text { Fraction } \\
\text { analyzed }\end{array}$} & \multicolumn{5}{|c|}{ 2/1 Ratio } \\
\hline & & $16: 0$ & $18: 0$ & $16: 1$ & $18: 1$ & $18: 2^{b}$ \\
\hline \multirow{2}{*}{ Fat free } & Lecithin & 0.03 & 0.0 & 1.1 & 2,0 & $\ldots$. \\
\hline & Triglyceride & 0.17 & 0.05 & 0.8 & 2.1 & $\ldots$ \\
\hline \multirow[t]{2}{*}{$\mathrm{t} 9, \mathrm{t} 12$} & Lecithin & 0.16 & 0.10 & 2.0 & 2.5 & 0.6 \\
\hline & 'Triglyceride & 0.27 & 0.25 & 1.3 & 1.8 & 0.5 \\
\hline \multirow[t]{2}{*}{$\mathrm{c9}, \mathrm{t} 12$} & Lecithin & 0.13 & 0.05 & 2.3 & 2.3 & 2.5 \\
\hline & Triglyceride & 0.12 & & 1.1 & 1.8 & 1.4 \\
\hline \multirow[t]{2}{*}{ c9,e12 } & Lecithin & 0.14 & 0.11 & 0.7 & 0.8 & 5.5 \\
\hline & Triglyceride & 0.15 & 0.5 & 1.0 & 1.6 & 4.9 \\
\hline
\end{tabular}

a Within the limits of detection in these experiments the eicosapolyenoates, $20: 3$ and $20: 4$, were located at the 2 position.

$\mathrm{b}$ The values in this column differ from those in the others, in that each pair represents a different acid which was fed. 
TABLE III

Composition of Rat Liver Lecithins and Triglycerides

\begin{tabular}{|c|c|c|c|c|c|c|c|c|c|}
\hline Diet & $\begin{array}{l}\text { Lipid } \\
\text { fraction }\end{array}$ & $18: 0$ & $16: 0$ & $16: 1$ & $18: 1$ & $18: 2^{a}$ & $18: 3^{a}$ & $20: 3^{a}$ & $20: 4^{a}$ \\
\hline \multirow[t]{2}{*}{ Fat free } & Lecithin & 15.6 & 24.1 & 8.5 & 29.1 & 1.7 & 0.5 & 14.9 & 2.2 \\
\hline & Triglyceride & 2.1 & 31.6 & 18.5 & 51.5 & & & & \\
\hline \multirow[t]{2}{*}{$\mathrm{t} 9, \mathrm{t} 12$} & Lecithin & 11.6 & 18.5 & 10.2 & 35.7 & 13.3 & 3.9 & 5.0 & 1.4 \\
\hline & Triglycenide & 3.8 & 28.6 & 13.2 & 49.2 & 5.7 & $\ldots$ & & \\
\hline \multirow[t]{2}{*}{$c 9, \mathrm{t} 12$} & Lecithin & 15.7 & 21.9 & 5.0 & 29.3 & 8.7 & & 7.4 & 12.0 \\
\hline & Triglyceride & 1.5 & 27.1 & 10.3 & 55.0 & 5.3 & 0.8 & $\ldots$ & \\
\hline \multirow[t]{2}{*}{$\mathrm{c} 9, \mathrm{c} 12$} & Lecithin & 19.2 & 20.9 & 1.5 & 9.2 & 14.6 & $\ldots$ & 1.5 & 33.0 \\
\hline & Triglyceride & 2.4 & 29.4 & 7.7 & 53.5 & 5.4 & $\ldots$ & & $\ldots \cdot$ \\
\hline
\end{tabular}

a Different geometrical isomers are present depending upon the dietary acid.

liver is known to produce other positional isomers through chain lengthening or shortening reactions. This process is recognized to be the source of vaccenate (12), and may be responsible for 8,11-octadecadienoate and 4,7,10,13eicosatetraenoate in the liver. The different acyltransferase specificities found in this paper suggest that future studies could show differences in acyltransferase activity for the different positional isomers (13) of the unsaturated acids.

The ratio of the enzyme-catalyzed rates of esterification at the 2- and 1-positions has been presented as a characteristic number for each acid. This ratio would predict the relative distribution in vivo of a fatty acid between the two positions of lecithin if the acyltransferases in the tissue were saturated with acyl-CoA and operating under the maximal conditions used for the in vitro experiments. This may often be a real situation since the reported levels of acyl-CoA in liver are at least 5-10 times higher than the $K_{m}$ observed in our enzymatic studies (2). However, it would also be useful to note that these data suggest that competitive effects could occur with a mixture of acids. For instance a mixture of the trans-9,cis-12- and trans9, trans-12-acids would give most of the trans-9, cis-12-isomer at the 2-position even though that acid has equal rates of esterification at both positions in a system where competition is not involved (Table I). Differences between the actual in vivo distributions and the enzymatic $2 / 1$ ratios given in this paper could be due to such competitive effects. The trans,trans supplemented diet, for instance, produced lipids that contained mostly palmitate, stearate and oleate. Thus the octadecadienoate had only oleate as a significant competitor at the 2position, whereas at the 1-position it competed with palmitate and stearate which both go well into that position. In such a situation, the $2 / 1$ ratio could be expected to be shifted to a value (0.6) slightly higher than that noted in the noncompeting spectrophotometrie assay system (0.21). Probably the most im- portant additional factor altering the in vivo distribution in these experiments is the widely varying content of eicosapolyenoic acids in the lecithins as a result of the unusual diets. These acids account for about 60 to 70,30 to 40 , and 10 to $15 \%$, of the acids at the 2-position of the phospholipid from animals of the cis-9, cis-12, the cis-9,trans-12, and trans-9,trans- 12 supplemented diets, respectively. It should be emphasized that the enzymatic studies used washed rat liver microsomes in hope that the collection of acyltransferases in this preparation would be representative of the acylating activity of the intact liver. The general agreement between the in vitro and in vivo results suggests that the enzymes studied may be the significant part of those enzymes controlling fatty acid distributions. Furthermore, the observed retention of the relative specific activities of these enzymes under the variety of dietary conditions mentioned earlier suggests that they are a stable characteristic of the normal liver cell. In addition to these results with rat liver, a very elose similarity in the observed acyltransferase $2 / 1$ ratios to the distributions found in vivo has also been recognized recently for pig liver lipids (14).

In the ease of those acids which have $2 / 1$ distribution ratios greater than 1, there is relatively little of the acid at the 1-position of lecithin. Therefore when the 1,2-diglyceride unit is converted metabolically to triglyceride, ineorporating such an acid into the 3-position would cause a marked percentage increase in its average content at the primary $(1-+3-)$ positions. This increase would then give a lower calculated $2 / 1$ ratio for that acid in the triglycerides than in the choline-phosphoglyeerides. On the other hand, the acids which are located predominantly at the primary position of leeithins would be predicted to exhibit less difference in the calculated $2 / 1$ ratios for the two different lipids. These considerations may be significant in interpreting the differences in the $2 / 1$ ratios seen in Table II.

Lipios, Vol. 1, No. 3 
At this time we cannot be certain whether the esterification at the 3 -position is random, less specifie than the acyl-CoA :phospholipid aeyltransferases, or specific for unsaturated acids. Nore careful experiments which differentiate between the 1 - and 3 -positions of a triglyceride should help distinguish between these possibilities. One such experimental method has been described by Brockerhoff (15) who has recently noted that asymmetrical fats have an excess of palmitate at the 1-position with more oleate at the 3-position (16). Further results of this type will be particularly useful in determining the selectivity of the enzymes catalyzing the esterification of the 3-position. 'These results will also allow a more precise comparison of the distributions of acids between the 1- and 2-positions of the triglycerides for comparison with those found in leeithins.

At the present, we can already see a considerable similarity in the positional distribution of acids in the diglyceride units of liver lecithins and triglycerides. It is thus somewhat ironic that the discovery of acyltransferases began with the recognition that the diglyceride unit of phospholipids is metabolically different in some respect from that of the triglycerides (17). This conclusion was based on different rates of isotopic tracer incorporation, and it led to the present concept of the acyl-CoA :phospholipid acyltransferases ineorporating fatty acids into lecithins without involving the diglycerides or triglycerides as intermediates. This metabolic difference has again been shown in the recent studies of stearie acid incorporation reported by Elofson (18). Comparison of the diglyceride radioactivity with that of the triglycerides and phosphoglycerides led Elofson to the conclusion that in vivo the absolute majority of labeled stearate in glycerolipids is incorporated via the acylCoA:phospholipid acyltransferase pathway. Thus the results with isotopic tracers indicate the differences in metabolic turnover rates of the ester bonds whereas the results of the positional analyses for each acid indicate that the 1,2-diglyceride units of lecithins and triglycerides are similar and may slowly equilibrate with each other. Such processes would allow the specificities of the acyl-CoA :acyl-GPC acyltransferases to be observed in both lecithins and triglycerides.

\section{ACKNOWLEDGMENT}

Supported in part by grants from the United States Public Health Service, AM-05310 and AM-04942.

\section{REFERENCES}

1. Lands, W. E. M, and P. Hart, J. Lipid Res. 5, 81 (1964).

2. Lands, W. E. M, and P. Hart, J. Biol. Chem. 240,1905 (1965)

3. Lands, W. E. M., JAOCS 42,465 (1965).

4. Seubert, W., Biochem. Prep. 7, 80 (1960).

5. Ellman, G. L., Arch. Biochem. Biophys. 82, 70 (1959).

6. Bartlett, G. R., J, Biol. Chem. 234, 466 (1959)

7. Hirschmann, H., J. Biol. Chem. 235, 2762 (1960).

8. Privett, O. S., and M. L. Blank, JAOOS 41,292 (1964).

9. Rouser, G., A. J. Bauman, G. Kritchersky, D. Heller and J. S. O'Brien, JAOCS 38, 544 (1961).

10. Robertson, A. F., and W. E. M. Lands, Biochemistry 1,804 (1962).

11. Mattson, F. H., and R. A. Volpenhein, J. Lipid Res, 2, 58 (1961).

12. Holloway, P. W., and S. J. Wakil, J. Biol, Chem. $239,2489(1964)$.

13. Klenk, $\mathrm{E}_{*}$, and G. Trchope, Z. Physiol. Chem. 334,193 (1963).

14. Lands, W, E, M., Ann, Rev, Biochem. 34, 313 (1965).

15. Brockerhoff, H., J. Lipid Res. 6, 10 (1965).

16. Brockerhoff, H., R. J. Hoyle, and N. Wolmark, Biochem. Biophys. Acta 116, 67 (1966).

17. Lands, W. E. M., J. Biol. Chem. 231, 883 (1958), 18. Elofson, J., Biochim. Biophys. Acta, in press.

[Received Jan. 10, 1966] 